\title{
Finiteness of selfinjective dimension for noetherian algebras
}

\author{
Mitsuo Hoshino and Hirotaka Koga
}

\begin{abstract}
We will study coherent modules of finite weak Gorenstein dimension and characterize noetherian algebras of finite selfinjective dimension in terms of weak Gorenstein dimension.
\end{abstract}

For an artin algebra $A$ we know from [9] that inj $\operatorname{dim} A=\operatorname{inj} \operatorname{dim} A^{\text {op }}<\infty$ if and only if every simple $X \in \bmod -A$ has finite Gorenstein dimension, where $A^{\mathrm{op}}$ is the opposite ring of $A$. Our main aim of this note is to extend this fact to noetherian algebras.

Let $R$ be a commutative noetherian local ring and $A$ a noetherian $R$-algebra, i.e., $A$ is a ring endowed with a ring homomorphism $R \rightarrow A$ whose image is contained in the center of $A$ and $A$ is finitely generated as an $R$-module. In order to state our main result, we have to recall the notion of weak Gorenstein dimension which was first introduced in [10]. A complex $X^{\bullet} \in \mathcal{D}^{\mathrm{b}}(\bmod -A)$ with $\sup \left\{i \mid \mathrm{H}^{i}\left(X^{\bullet}\right) \neq 0\right\}=d<\infty$ is said to have finite weak Gorenstein dimension if $\mathbf{R H o m}_{A}^{\bullet}\left(X^{\bullet}, A\right) \in \mathcal{D}^{\mathrm{b}}\left(\bmod -A^{\text {op }}\right), \mathrm{H}^{i}\left(\eta_{X} \bullet\right)$ is an isomorphism for all $i<d$ and $\mathrm{H}^{d}\left(\eta_{X^{\bullet}}\right)$ is a monomorphism, where $\eta_{X} \bullet: X^{\bullet} \rightarrow \mathbf{R H o m}_{A^{\text {op }}}\left(\mathbf{R H o m}_{A}^{\bullet}\left(X^{\bullet}, A\right), A\right)$ denotes the canonical homomorphism (see [11]). For a module $X \in \bmod -A$ of finite weak Gorenstein dimension we set $\hat{\mathrm{G}}-\operatorname{dim} X=\sup \left\{i \mid \operatorname{Ext}_{A}^{i}(X, A) \neq 0\right\}$ unless $X=0$ and $\hat{\mathrm{G}}$-dim $X=0$ if $X=0$. Also, we set $\hat{\mathrm{G}}$-dim $X=\infty$ if $X$ does not have finite weak Gorenstein dimension. Then $\hat{\mathrm{G}}$-dim $X$ is called the weak Gorenstein dimension of $X \in \bmod -A$. Denote by $\mathrm{G}$-dim $X$ the Gorenstein dimension of $X \in \bmod -A$ (see [2] and [11]). Then $\hat{\mathrm{G}}$-dim $X \leq \mathrm{G}$-dim $X$ for all $X \in \bmod -A$ : $\hat{\mathrm{G}}$-dim $X=\mathrm{G}$-dim $X$ if $\mathrm{G}$-dim $X<\infty$, but $\hat{\mathrm{G}}$-dim $X \neq \mathrm{G}$-dim $X$ in general (see [10, Example 4.3]).

A noetherian $R$-algebra $A$ is said to satisfy the condition $(\mathrm{G})$ if the following equivalent conditions are satisfied: (1) $\hat{\mathrm{G}}$-dim $X<\infty$ for all simple $X \in \bmod -A$; and $(2) \hat{\mathrm{G}}-\operatorname{dim} A / \operatorname{rad}(A)<\infty$. Then $\operatorname{inj} \operatorname{dim} A=\operatorname{inj} \operatorname{dim} A^{\text {op }}<\infty$ if and only if $A_{\mathfrak{p}}$ satisfies the condition $(\mathrm{G})$ for all $\mathfrak{p} \in \operatorname{Supp}_{R}(A)$ (Theorem 3.7). This is our main result of this note, the proof of which is based on the fact that $\operatorname{Hom}_{R}\left(A, E_{R}(R / \mathfrak{m})\right) \in \operatorname{Mod}-A$ is an injective cogenerator and isomorphic to

2010 Mathematics Subject Classification. Primary 16E10; Secondary 16E35, 18 E30.

Key words. Gorenstein algebra, Gorenstein dimension, Derived category. 
a direct limit of modules of finite length, where $\mathfrak{m}$ is the maximal ideal of $R$. In case $A$ is a local ring, we will show that for any $d \geq 0$ the following are equivalent: (1) inj $\operatorname{dim} A=\operatorname{inj} \operatorname{dim} A^{\text {op }}=d$; (2) inj $\operatorname{dim} A=\operatorname{depth} A=d$; and (3) $\hat{\mathrm{G}}$-dim $A / \operatorname{rad}(A)=d$ (Theorem 4.7). It should be noted that if inj $\operatorname{dim} A=$ depth $A<\infty$ then $A$ is a Gorenstein algebra in the sense of Goto and Nishida [7] (cf. Lemma 4.2).

This note is organized as follows. In Section 1, we will recall several basic facts. In Section 2, we will recall the notion of weak Gorenstein dimension and study coherent modules of finite weak Gorenstein dimension. We will show that the class of such modules is closed under extensions (Lemma 2.7) and provide a sufficient condition for $X \in \bmod -A$ with $\operatorname{RHom}_{A}^{\bullet}(X, A) \in \mathcal{D}^{\mathrm{b}}\left(\bmod -A^{\text {op }}\right)$ to have finite weak Gorenstein dimension (Proposition 2.8). In Section 3, we will study noetherian algebras of finite selfinjective dimension and prove our main theorem. In Section 4, we will characterize local noetherian algebras of finite selfinjective dimension in terms of weak Gorenstein dimension. Also, we will provide several examples showing what rich properties local noetherian algebras of finite selfinjective dimension enjoy.

\section{Preliminaries}

For a $\operatorname{ring} A$ we denote by $\operatorname{rad}(A)$ the Jacobson radical of $A$. We denote by Mod- $A$ the category of right $A$-modules, by $\bmod -A$ the full subcategory of Mod- $A$ consisting of finitely presented modules and by $\mathcal{P}_{A}$ the full subcategory of mod- $A$ consisting of projective modules. For each $X \in \operatorname{Mod}-A$ we denote by $E_{A}(X)$ its injective envelope and by $\operatorname{Ann}_{A}(X)$ its annihilator ideal. Left $A$ modules are considered as right $A^{\mathrm{op}}$-modules, where $A^{\text {op }}$ denotes the opposite ring of $A$. In particular, we denote by inj $\operatorname{dim} A$ (resp., inj $\operatorname{dim} A^{\mathrm{op}}$ ) the injective dimension of $A$ as a right (resp., left) $A$-module and by $\operatorname{Hom}_{A}(-,-)$ (resp., $\left.\operatorname{Hom}_{A^{\mathrm{op}}}(-,-)\right)$ the set of homomorphisms in Mod- $A$ (resp., Mod- $A^{\mathrm{op}}$ ). Sometimes, we use the notation $X_{A}$ (resp., ${ }_{A} X$ ) to stress that the module considered is a right (resp., left) $A$-module.

In this note, complexes are cochain complexes and modules are considered as complexes concentrated in degree zero. We denote by $\mathcal{K}(\operatorname{Mod}-A)$ the homotopy category of complexes over $\operatorname{Mod}-A$, by $\mathcal{K}^{-}\left(\mathcal{P}_{A}\right)\left(\operatorname{resp} ., \mathcal{K}^{\mathrm{b}}\left(\mathcal{P}_{A}\right)\right.$ ) the full triangulated subcategory of $\mathcal{K}(\operatorname{Mod}-A)$ consisting of bounded above (resp., bounded) complexes over $\mathcal{P}_{A}$ and by $\mathcal{K}^{-, \mathrm{b}}\left(\mathcal{P}_{A}\right)$ the full triangulated subcategory of $\mathcal{K}^{-}\left(\mathcal{P}_{A}\right)$ consisting of complexes with bounded cohomology. We denote by $\mathcal{D}(\operatorname{Mod}-A)$ the derived category of complexes over Mod- $A$. Also, we denote by $\operatorname{Hom}_{A}^{\bullet}(-,-)$ (resp., $-\otimes \bullet-$ ) the associated single complex of the double hom (resp., tensor) complex and by $\mathbf{R H o m}_{A}^{\bullet}(-, A)$ the right derived functor of $\operatorname{Hom}_{A}^{\bullet}(-, A)$. We refer to [3], [8] and [13] for basic results in the theory of derived categories.

In this section, we will recall several definitions and basic facts.

Definition 1.1. For a complex $X^{\bullet}$ and an integer $n \in \mathbb{Z}$, we denote by $\mathrm{Z}^{n}\left(X^{\bullet}\right)$, $\mathrm{Z}^{\prime n}\left(X^{\bullet}\right)$ and $\mathrm{H}^{n}\left(X^{\bullet}\right)$ the $n$th cycle, the $n$th cocycle and the $n$th cohomology, 
respectively, and define the following truncations:

$$
\begin{aligned}
& \sigma_{\leq n}\left(X^{\bullet}\right): \cdots \rightarrow X^{n-2} \rightarrow X^{n-1} \rightarrow \mathrm{Z}^{n}\left(X^{\bullet}\right) \rightarrow 0 \rightarrow \cdots, \\
& \sigma_{\geq n}^{\prime}\left(X^{\bullet}\right): \cdots \rightarrow 0 \rightarrow \mathrm{Z}^{\prime n}\left(X^{\bullet}\right) \rightarrow X^{n+1} \rightarrow X^{n+2} \rightarrow \cdots,
\end{aligned}
$$

Definition $1.2([5])$. A module $X \in \operatorname{Mod}-A$ is said to be coherent if it is finitely generated and every finitely generated submodule of it is finitely presented. A ring $A$ is said to be left (resp., right) coherent if it is coherent as a left (resp., right) $A$-module.

Throughout the first two sections, $A$ is a left and right coherent ring. Note that $\bmod -A$ consists of coherent modules and is a thick abelian subcategory of Mod- $A$ in the sense of [8], i.e., it holds true for any short exact sequence in $\operatorname{Mod}-A$ that if any two terms are in $\bmod -A$ then the remaining term also is in $\bmod -A$.

We denote by $\mathcal{D}^{\mathrm{b}}(\bmod -A)$ the full triangulated subcategory of $\mathcal{D}(\operatorname{Mod}-A)$ consisting of complexes over mod- $A$ with bounded cohomology.

Definition $1.3([8])$. A complex $X^{\bullet} \in \mathcal{D}^{\mathrm{b}}(\bmod -A)$ is said to have finite projective dimension if $\operatorname{Hom}_{\mathcal{D}(\operatorname{Mod}-A)}\left(X^{\bullet}[i],-\right)$ vanishes on $\bmod -A$ for $i \ll 0$. We denote by $\mathcal{D}^{\mathrm{b}}(\bmod -A)_{\text {fpd }}$ the épaisse subcategory of $\mathcal{D}^{\mathrm{b}}(\bmod -A)$ consisting of complexes of finite projective dimension.

Note that the canonical functor $\mathcal{K}(\operatorname{Mod}-A) \rightarrow \mathcal{D}(\operatorname{Mod}-A)$ gives rise to equivalences of triangulated categories

$$
\mathcal{K}^{-, \mathrm{b}}\left(\mathcal{P}_{A}\right) \stackrel{\sim}{\rightarrow} \mathcal{D}^{\mathrm{b}}(\bmod -A) \text { and } \quad \mathcal{K}^{\mathrm{b}}\left(\mathcal{P}_{A}\right) \stackrel{\sim}{\rightarrow} \mathcal{D}^{\mathrm{b}}(\bmod -A)_{\text {fpd }} .
$$

We denote by $D(-)$ both $\mathbf{R H o m}_{A}^{\bullet}(-, A)$ and $\mathbf{R} \operatorname{Hom}_{A^{\text {op }}}(-, A)$. There exists a bifunctorial isomorphism

$$
\theta_{M}, X \bullet: \operatorname{Hom}_{\mathcal{D}\left(\operatorname{Mod}-A^{\text {op }}\right)}\left(M^{\bullet}, D X^{\bullet}\right) \stackrel{\sim}{\rightarrow} \operatorname{Hom}_{\mathcal{D}(\operatorname{Mod}-A)}\left(X^{\bullet}, D M^{\bullet}\right)
$$

for $X^{\bullet} \in \mathcal{D}(\operatorname{Mod}-A)$ and $M^{\bullet} \in \mathcal{D}\left(\operatorname{Mod}-A^{\text {op }}\right)$. For each $X^{\bullet} \in \mathcal{D}(\operatorname{Mod}-A)$ we set

$$
\eta_{X} \bullet=\theta_{D X} \bullet, X^{\bullet}\left(\operatorname{id}_{D X} \bullet\right): X^{\bullet} \rightarrow D^{2} X^{\bullet}=D\left(D X^{\bullet}\right) .
$$

Definition 1.4. A complex $X^{\bullet} \in \mathcal{D}^{\mathrm{b}}(\bmod -A)$ is said to have bounded dual cohomology if $D X^{\bullet} \in \mathcal{D}^{\mathrm{b}}\left(\bmod -A^{\mathrm{op}}\right)$. We denote by $\mathcal{D}^{\mathrm{b}}(\bmod -A)_{\text {bdh }}$ the full triangulated subcategory of $\mathcal{D}^{\mathrm{b}}(\bmod -A)$ consisting of complexes with bounded dual cohomology.

Definition $1.5([2]$ and $[11])$. A complex $X^{\bullet} \in \mathcal{D}^{\mathrm{b}}\left(\bmod -A^{\mathrm{op}}\right)_{\mathrm{bdh}}$ is said to have finite Gorenstein dimension if $\eta_{X}$ • is an isomorphism. We denote by $\mathcal{D}^{\mathrm{b}}(\bmod -A)_{\mathrm{fGd}}$ the full triangulated subcategory of $\mathcal{D}^{\mathrm{b}}(\bmod -A)$ consisting of complexes of finite Gorenstein dimension. 
For a module $X \in \mathcal{D}^{\mathrm{b}}(\bmod -A)_{\mathrm{fGd}}$ we set

$$
\text { G-dim } X=\sup \left\{i \geq 0 \mid \operatorname{Ext}_{A}^{i}(X, A) \neq 0\right\}
$$

unless $X=0$ and G-dim $X=0$ if $X=0$. Also, we set G-dim $X=\infty$ unless $X \in \mathcal{D}^{\mathrm{b}}(\bmod -A)_{\mathrm{fGd}}$. Then G-dim $X$ is called the Gorenstein dimension of $X \in \bmod -A$. We denote by $\mathcal{G}_{A}$ the full additive subcategory of mod- $A$ consisting of modules of Gorenstein dimension zero.

Remark 1.6. A module $X \in \bmod -A$ has Gorenstein dimension zero if and only if $X$ is reflexive, i.e., the canonical homomorphism

$$
X \rightarrow \operatorname{Hom}_{A^{\text {op }}}\left(\operatorname{Hom}_{A}(X, A), A\right), x \mapsto(f \mapsto f(x))
$$

is an isomorphism and $\operatorname{Ext}_{A}^{i}(X, A)=\operatorname{Ext}_{A^{\text {op }}}^{i}\left(\operatorname{Hom}_{A}(X, A), A\right)=0$ for $i \neq 0$.

Remark 1.7. The following hold.

(1) $\mathcal{D}^{\mathrm{b}}(\bmod -A)_{\mathrm{fpd}} \subseteq \mathcal{D}^{\mathrm{b}}(\bmod -A)_{\mathrm{fGd}} \subseteq \mathcal{D}^{\mathrm{b}}(\bmod -A)_{\mathrm{bdh}}$ and $\mathcal{P}_{A} \subseteq \mathcal{G}_{A}$.

(2) The pair of functors $\mathbf{R H o m}_{A}^{\bullet}(-, A)$ and $\mathbf{R H o m}_{A^{\text {op }}}(-, A)$ defines a duality between $\mathcal{D}^{\mathrm{b}}(\bmod -A)_{\mathrm{fGd}}$ and $\mathcal{D}^{\mathrm{b}}\left(\bmod -A^{\mathrm{op}}\right)_{\mathrm{fGd}}$ and a duality between $\mathcal{D}^{\mathrm{b}}(\bmod -A)_{\mathrm{fpd}}$ and $\mathcal{D}^{\mathrm{b}}\left(\bmod -A^{\mathrm{op}}\right)_{\mathrm{fpd}}$.

(3) The pair of functors $\operatorname{Hom}_{A}(-, A)$ and $\operatorname{Hom}_{A^{\text {op }}}(-, A)$ defines a duality between $\mathcal{G}_{A}$ and $\mathcal{G}_{A^{\text {op }}}$ and a duality between $\mathcal{P}_{A}$ and $\mathcal{P}_{A^{\text {op }}}$.

Lemma 1.8. Let $A \rightarrow I^{\bullet}$ be a minimal injective resolution in Mod-A. Assume that flat $\operatorname{dim} I^{n}<\infty$ for all $n \geq 0$ and that for any nonzero $X \in \bmod -A$ there exists $n \geq 0$ such that $\operatorname{Hom}_{A}\left(X, I^{n}\right) \neq 0$. Then $X^{\bullet} \in \mathcal{D}^{\mathrm{b}}(\bmod -A)_{\mathrm{fGd}}$ for all $X^{\bullet} \in \mathcal{D}^{\mathrm{b}}(\bmod -A)_{\text {bdh }}$.

Proof. Let $X^{\bullet} \in \mathcal{D}^{\mathrm{b}}(\bmod -A)_{\mathrm{bdh}}$ and take a quasi-isomorphism $P^{\bullet} \rightarrow X^{\bullet}$ with $P^{\bullet} \in \mathcal{K}^{-, \mathrm{b}}\left(\mathcal{P}_{A}\right)$. Let $Q^{\bullet} \rightarrow \operatorname{Hom}_{A}^{\bullet}\left(P^{\bullet}, A\right)$ be a quasi-isomorphism with $Q^{\bullet} \in$ $\mathcal{K}^{-, \mathrm{b}}\left(\mathcal{P}_{A^{\text {op }}}\right)$ and $Z^{\bullet}$ its mapping cone. Then we have a distinguished triangle in $\mathcal{K}(\operatorname{Mod}-A)$

$$
\operatorname{Hom}_{A^{\text {op }}}^{\bullet}\left(Z^{\bullet}, A\right) \rightarrow P^{\bullet} \rightarrow \operatorname{Hom}_{A^{\text {op }}}^{\bullet}\left(Q^{\bullet}, A\right) \rightarrow .
$$

It suffices to show that $\mathrm{H}^{i}\left(\operatorname{Hom}_{A^{\text {op }}}^{\bullet}\left(Z^{\bullet}, A\right)\right)=0$ for all $i \in \mathbb{Z}$. Since $\mathrm{H}^{i}\left(Z^{\bullet}\right)=0$ for all $i \in \mathbb{Z}$, it follows that $\mathrm{H}^{i}\left(\operatorname{Hom}_{A^{\text {op }}}^{\bullet}\left(Z^{\bullet}, A\right)\right) \cong \operatorname{Ext}_{A^{\text {op }}}^{1}\left(\mathrm{Z}^{\prime-i+1}\left(Z^{\bullet}\right), A\right) \cong$ $\operatorname{Ext}_{A^{\text {op }}}\left(Z^{\prime-i+j}\left(Z^{\bullet}\right), A\right)$ for all $i \in \mathbb{Z}$ and $j>0$. Also, for any $i \in \mathbb{Z}$ and $n \geq 0$ we have $\operatorname{Hom}_{A}\left(\operatorname{Ext}_{A^{\text {op }}}^{j}\left(\mathrm{Z}^{\prime-i+j}\left(Z^{\bullet}\right), A\right), I^{n}\right) \cong \operatorname{Tor}_{j}^{A}\left(I^{n}, \mathrm{Z}^{\prime-i+j}\left(Z^{\bullet}\right)\right)=0$ for $j \gg 0$. Thus $\mathrm{H}^{i}\left(\operatorname{Hom}_{A^{\text {op }}}\left(Z^{\bullet}, A\right)\right)=0$ for all $i \in \mathbb{Z}$.

Remark 1.9. The assumptions in the lemma above are fulfilled if $\operatorname{Ext}_{A^{\text {op }}}^{i}(-, A)$ vanishes on $\bmod -A^{\text {op }}$ for $i \gg 0$.

Proof. For any injective $I \in \operatorname{Mod}-A$ and $M \in \bmod -A^{\text {op }}$ we have $\operatorname{Tor}_{i}^{A}(I, M) \cong$ $\operatorname{Hom}_{A}\left(\operatorname{Ext}_{A \text { op }}^{i}(M, A), I\right)=0$ for $i \gg 0$. Also, for any $X \in \bmod -A$ with $\operatorname{Hom}_{A}\left(X, I^{n}\right)=0$ for all $n \geq 0$, we have $\operatorname{Ext}_{A}^{n}(X, A)=0$ for all $n \geq 0$ and hence, taking a projective resolution $P^{\bullet} \rightarrow X$ in mod- $A$, we have $X \cong$ $\operatorname{Ext}_{A^{\text {op }}}^{i}\left(\mathrm{Z}^{\prime i}\left(\operatorname{Hom}_{A}^{\bullet}\left(P^{\bullet}, A\right)\right), A\right)=0$ for $i \gg 0$. 


\section{Weak Gorenstein dimension}

In this section, we will study coherent modules of finite weak Gorenstein dimension, the notion of which was first introduced in [10].

Definition 2.1. We denote by $\hat{\mathcal{G}}_{A}$ the full additive subcategory of mod- $A$ consisting of modules $X \in \bmod -A$ with $\operatorname{Ext}_{A}^{i}(X, A)=0$ for $i \neq 0$.

Definition 2.2. A complex $X^{\bullet} \in \mathcal{D}^{\mathrm{b}}(\bmod -A)$ with $\sup \left\{i \mid \mathrm{H}^{i}\left(X^{\bullet}\right) \neq 0\right\}=$ $d<\infty$ is said to have finite weak Gorenstein dimension if $X^{\bullet} \in \mathcal{D}^{\mathrm{b}}(\bmod -A)_{\mathrm{bdh}}$, $\mathrm{H}^{i}\left(\eta_{X^{\bullet}}\right)$ is an isomorphism for all $i<d$ and $\mathrm{H}^{d}\left(\eta_{X^{\bullet}}\right)$ is a monomorphism.

For a module $X \in \bmod -A$ of finite weak Gorenstein dimension we set

$$
\hat{\mathrm{G}}-\operatorname{dim} X=\sup \left\{i \mid \operatorname{Ext}_{A}^{i}(X, A) \neq 0\right\}
$$

unless $X=0$ and $\hat{\mathrm{G}}$-dim $X=0$ if $X=0$. Also, we set $\hat{\mathrm{G}}$-dim $X=\infty$ if $X$ does not have finite weak Gorenstein dimension. Then $\hat{\mathrm{G}}$-dim $X$ is called the weak Gorenstein dimension of $X \in \bmod -A$.

Remark 2.3. For any $X \in \bmod -A$ the following hold.

(1) $\hat{\mathrm{G}}$-dim $X=0$ if and only if $X$ is embedded in some $P \in \mathcal{P}_{A}$ and $X \in \hat{\mathcal{G}}_{A}$, i.e., the canonical homomorphism

$$
X \rightarrow \operatorname{Hom}_{A^{\mathrm{op}}}\left(\operatorname{Hom}_{A}(X, A), A\right), x \mapsto(f \mapsto f(x))
$$

is a monomorphism and $\operatorname{Ext}_{A}^{i}(X, A)=0$ for $i \neq 0$.

(2) If G-dim $X=d<\infty$ then $\hat{\mathrm{G}}-\operatorname{dim} X=d$.

(3) If $\hat{\mathrm{G}}$-dim $X=d<\infty$ then $\hat{\mathrm{G}}$-dim $X^{\prime} \leq d$ for all $X^{\prime} \in \operatorname{add}(X)$, the full additive subcategory of $\bmod -A$ consisting of direct summands of finite direct sums of copies of $X$.

Proof. (1) "Only if" part. Since $X \in \hat{\mathcal{G}}_{A}, \operatorname{Hom}_{A}(X, A) \cong D X$ in $\mathcal{D}^{\mathrm{b}}\left(\bmod -A^{\text {op }}\right)$. Take a projective resolution $Q^{\bullet} \rightarrow \operatorname{Hom}_{A}(X, A)$ in $\bmod -A^{\text {op }}$. Then $D^{2} X \cong$ $\operatorname{Hom}_{A^{\text {op }}}\left(Q^{\bullet}, A\right)$ in $\mathcal{D}^{\mathrm{b}}(\bmod -A)$. Since $\mathrm{H}^{0}\left(\eta_{X}\right)$ is a monomorphism, $X$ is embedded in $\operatorname{Hom}_{A^{\mathrm{op}}}\left(Q^{0}, A\right) \in \mathcal{P}_{A}$.

"If" part. We have an exact sequence $0 \rightarrow X \stackrel{\mu}{\rightarrow} P \rightarrow Y \rightarrow 0$ in mod- $A$ with $P \in \mathcal{P}_{A}$ and a distinguished triangle $D^{2} X \stackrel{D^{2} \mu}{\longrightarrow} D^{2} P \rightarrow D^{2} Y \rightarrow$ in $\mathcal{D}^{\mathrm{b}}(\bmod -A)$. Since $X \in \hat{\mathcal{G}}_{A}, \operatorname{Ext}_{A}^{i+1}(Y, A) \cong \operatorname{Ext}_{A}^{i}(X, A)=0$ for $i>0$. Take a projective resolution $Q^{\bullet} \rightarrow D Y$ in $\mathcal{D}^{\mathrm{b}}\left(\bmod -A^{\text {op }}\right)$ with $Q^{i}=0$ for $i>1$. By the long exact cohomology sequence we have $\mathrm{H}^{-i}\left(D^{2} X\right) \cong \mathrm{H}^{-i-1}\left(D^{2} Y\right) \cong$ $\mathrm{H}^{-i-1}\left(\operatorname{Hom}_{A^{\text {op }}}\left(Q^{\bullet}, A\right)\right)=0$ for $i>0$. Finally, $\mathrm{H}^{0}\left(D^{2} \mu\right) \circ \mathrm{H}^{0}\left(\eta_{X}\right)=\mathrm{H}^{0}\left(\eta_{P}\right) \circ \mu$ is a monomorphism and so is $\mathrm{H}^{0}\left(\eta_{X}\right)$.

(2) Obvious.

(3) Since $X^{\prime} \in \operatorname{add}(X), D X^{\prime}$ is a direct summand of a finite direct sum of copies of $D X$ and $D^{2} X^{\prime}$ is a direct summand of a finite direct sum of copies of $D^{2} X$, so that $\mathrm{H}^{i+d}\left(D X^{\prime}\right)=0$ and $\mathrm{H}^{-i}\left(D^{2} X^{\prime}\right)=0$ for $i>0$. Denoting by $\iota$ the split monomorphism $X^{\prime} \rightarrow \oplus^{n} X, \mathrm{H}^{0}\left(D^{2} \iota\right) \circ \mathrm{H}^{0}\left(\eta_{X^{\prime}}\right)=\mathrm{H}^{0}\left(\eta_{\oplus^{n} X}\right) \circ \iota$ is a monomorphism and so is $\mathrm{H}^{0}\left(\eta_{X^{\prime}}\right)$. 
Remark 2.4. In general, $\hat{\mathrm{G}}$-dim $X \neq \mathrm{G}$-dim $X$ (see [10, Example 4.3]).

Proposition 2.5 ([10, Proposition 2.7]). A complex $X^{\bullet} \in \mathcal{D}^{\mathrm{b}}(\bmod -A)$ with $\sup \left\{i \mid \mathrm{H}^{i}\left(X^{\bullet}\right) \neq 0\right\}=d<\infty$ has finite weak Gorenstein dimension if and only if there exists a distinguished triangle in $\mathcal{D}^{\mathrm{b}}(\bmod -A)$

$$
X^{\bullet} \rightarrow Y^{\bullet} \rightarrow Z[-d] \rightarrow
$$

with $Y^{\bullet} \in \mathcal{K}^{\mathrm{b}}\left(\mathcal{P}_{A}\right), Y^{i}=0$ for $i>d$, and $Z \in \hat{\mathcal{G}}_{A}$.

Corollary 2.6 ([10, Corollary 2.8]). For any $X \in \bmod -A$ with $\hat{\mathrm{G}}$-dim $X<\infty$ there exists an exact sequence $0 \rightarrow X \rightarrow Y \rightarrow Z \rightarrow 0$ in $\bmod -A$ with $\hat{\mathrm{G}}$-dim $X=$ proj $\operatorname{dim} Y$ and $Z \in \hat{\mathcal{G}}_{A}$.

The corollary above is a generalization of [6, Lemma 2.17].

Lemma 2.7. For any exact sequence $0 \rightarrow X \rightarrow Y \rightarrow Z \rightarrow 0$ in mod- $A$ the following hold.

(1) If $\hat{\mathrm{G}}-\operatorname{dim} Z<\infty$, then $\hat{\mathrm{G}}-\operatorname{dim} X<\infty$ if and only if $\hat{\mathrm{G}}-\operatorname{dim} Y<\infty$.

(2) If $\hat{\mathrm{G}}-\operatorname{dim} Y<\infty$, then $\hat{\mathrm{G}}-\operatorname{dim} X<\infty$ if and only if $Z \in \mathcal{D}^{\mathrm{b}}(\bmod -A)_{\mathrm{bdh}}$ and $\mathrm{H}^{i}\left(D^{2} Z\right)=0$ for $i<-1$.

(3) If $\hat{\mathrm{G}}-\operatorname{dim} X<\infty$ and $\mathrm{H}^{0}\left(\eta_{X}\right)$ is an isomorphism, then $\hat{\mathrm{G}}-\operatorname{dim} Y<\infty$ if and only if $\hat{\mathrm{G}}-\operatorname{dim} Z<\infty$.

Proof. We have a distinguished triangle $D Z \rightarrow D Y \rightarrow D X \rightarrow$ in $\mathcal{D}\left(\right.$ Mod- $-A^{\text {op }}$ ) and a distinguished triangle $D^{2} X \rightarrow D^{2} Y \rightarrow D^{2} Z \rightarrow$ in $\mathcal{D}(\operatorname{Mod}-A)$.

(1) Since $Z \in \mathcal{D}^{\mathrm{b}}(\bmod -A)_{\mathrm{bdh}}, X \in \mathcal{D}^{\mathrm{b}}(\bmod -A)_{\mathrm{bdh}}$ if and only if $Y \in$ $\mathcal{D}^{\mathrm{b}}(\bmod -A)_{\text {bdh }}$. Also, since $\mathrm{H}^{i}\left(D^{2} Z\right)=0$ for $i<0$, by the long exact cohomology sequence we have $\mathrm{H}^{i}\left(D^{2} X\right) \cong \mathrm{H}^{i}\left(D^{2} Y\right)$ for $i<0$ and a commutative diagram with exact rows

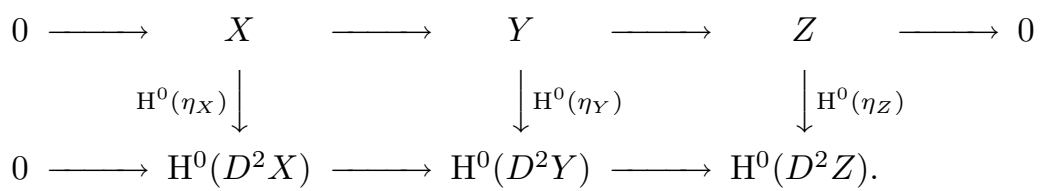

It follows by Snake lemma that $\operatorname{Ker} \mathrm{H}^{0}\left(\eta_{X}\right) \cong \operatorname{Ker} \mathrm{H}^{0}\left(\eta_{Y}\right)$.

(2) Since $Y \in \mathcal{D}^{\mathrm{b}}(\bmod -A)_{\mathrm{bdh}}, X \in \mathcal{D}^{\mathrm{b}}(\bmod -A)_{\mathrm{bdh}}$ if and only if $Z \in$ $\mathcal{D}^{\mathrm{b}}(\bmod -A)_{\mathrm{bdh}}$. Also, since $\mathrm{H}^{i}\left(D^{2} Y\right)=0$ for $i<0$, by the long exact cohomology sequence we have $\mathrm{H}^{i-1}\left(D^{2} Z\right) \cong \mathrm{H}^{i}\left(D^{2} X\right)$ for $i<0$. Finally, denoting by $\mu$ the monomorphism $X \rightarrow Y, \mathrm{H}^{0}\left(D^{2} \mu\right) \circ \mathrm{H}^{0}\left(\eta_{X}\right)=\mathrm{H}^{0}\left(\eta_{Y}\right) \circ \mu$ is a monomorphism and so is $\mathrm{H}^{0}\left(\eta_{X}\right)$.

(3) The "if" part follows by (1) above. Assume that $\hat{\mathrm{G}}$-dim $Y<\infty$. Since $X, Y \in \mathcal{D}^{\mathrm{b}}(\bmod -A)_{\mathrm{bdh}}, Z \in \mathcal{D}^{\mathrm{b}}(\bmod -A)_{\mathrm{bdh}}$. Also, by the long exact cohomology sequence we have $\mathrm{H}^{i}\left(D^{2} Z\right)=0$ for $i<-1$ and an exact sequence

$$
0 \rightarrow \mathrm{H}^{-1}\left(D^{2} Z\right) \rightarrow \mathrm{H}^{0}\left(D^{2} X\right) \rightarrow \mathrm{H}^{0}\left(D^{2} Y\right) .
$$


Since $\mathrm{H}^{0}\left(\eta_{X}\right)$ an isomorphism, and since $\mathrm{H}^{0}\left(\eta_{Y}\right)$ is a monomorphism, it follows that $\mathrm{H}^{0}\left(D^{2} X\right) \rightarrow \mathrm{H}^{0}\left(D^{2} Y\right)$ is a monomorphism and $\mathrm{H}^{-1}\left(D^{2} Z\right)=0$, so that we have a commutative diagram with exact rows

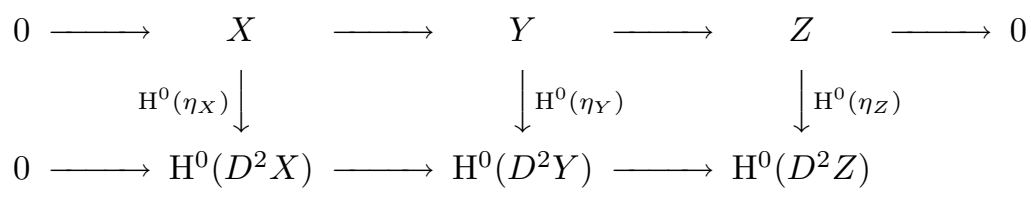

and by Snake lemma Ker $\mathrm{H}^{0}\left(\eta_{Z}\right) \cong \operatorname{Ker} \mathrm{H}^{0}\left(\eta_{Y}\right)=0$.

Proposition 2.8. Let $X \in \bmod -A$ with $\sup \left\{i \mid \operatorname{Ext}_{A}^{i}(X, A) \neq 0\right\}=d<\infty$. Let $P^{\bullet} \rightarrow X$ be a projective resolution in $\bmod -A$ and $M=\mathrm{Z}^{\prime d+1}\left(\operatorname{Hom}_{A}^{\bullet}\left(P^{\bullet}, A\right)\right)$. Then the following hold.

(1) $\hat{\mathrm{G}}-\operatorname{dim} X<\infty$ if and only if $\operatorname{Ext}_{A^{\mathrm{op}}}^{i}(M, A)=0$ for $1 \leq i \leq d+1$.

(2) $\operatorname{Ext}_{A^{\text {op }}}^{1}(M, A)=0$ unless $d=0$.

(3) If $\operatorname{Ext}_{A^{\mathrm{op}}}^{j}\left(\operatorname{Ext}_{A}^{i}(X, A), A\right)=0$ for $0 \leq j<i \leq d$ then $\operatorname{Ext}_{A^{\mathrm{op}}}^{i}(M, A)=0$ for $1 \leq i \leq d$ and $\operatorname{Ext}_{A^{\text {op }}}^{d+1}(M, A) \cong \operatorname{Ker}^{0}\left(\eta_{X}\right)$.

Proof. Let $Q^{\bullet} \rightarrow \operatorname{Hom}_{A}^{\bullet}\left(P^{\bullet}, A\right)$ be a quasi-isomorphism with $Q^{\bullet} \in \mathcal{K}^{-, \mathrm{b}}\left(\mathcal{P}_{A^{\text {op }}}\right)$, $Q^{i}=0$ for $i>d$, and $Z^{\bullet}$ its mapping cone. Then $\mathrm{H}^{i}\left(Z^{\bullet}\right)=0$ for all $i \in \mathbb{Z}$ and $\mathrm{Z}^{\prime d+1}\left(Z^{\bullet}\right) \cong M$, so that $\mathrm{H}^{-i}\left(\operatorname{Hom}_{A^{\text {op }}}\left(Z^{\bullet}, A\right)\right) \cong \operatorname{Ext}_{A^{\text {op }}}^{1}\left(\mathrm{Z}^{\prime i+1}\left(Z^{\bullet}\right), A\right) \cong$ $\operatorname{Ext}_{A^{\mathrm{op}}}^{d+1-i}(M, A)$ for $0 \leq i \leq d$. On the other hand, we have a distinguished triangle in $\mathcal{K}(\operatorname{Mod}-A)$

$$
\operatorname{Hom}_{A^{\text {op }}}^{\bullet}\left(Z^{\bullet}, A\right) \rightarrow P^{\bullet} \stackrel{\eta}{\rightarrow} \operatorname{Hom}_{A^{\text {op }}}^{\bullet}\left(Q^{\bullet}, A\right) \rightarrow
$$

and, since $\mathrm{H}^{-i}\left(P^{\bullet}\right)=0$ for $i>0$, by the long exact cohomology sequence we have $\mathrm{H}^{-i-1}\left(\operatorname{Hom}_{A^{\text {op }}}\left(Q^{\bullet}, A\right)\right) \stackrel{\sim}{\rightarrow} \mathrm{H}^{-i}\left(\operatorname{Hom}_{A^{\text {op }}}\left(Z^{\bullet}, A\right)\right)$ for $i>0$ and an exact sequence

$$
0 \rightarrow \mathrm{H}^{-1}\left(\operatorname{Hom}_{A^{\text {op }}}^{\bullet}\left(Q^{\bullet}, A\right)\right) \rightarrow \mathrm{H}^{0}\left(\operatorname{Hom}_{A^{\text {op }}}^{\bullet}\left(Z^{\bullet}, A\right)\right) \rightarrow \operatorname{Ker}^{0}(\eta) \rightarrow 0 .
$$

Now, since $\mathrm{H}^{-i}\left(\operatorname{Hom}_{A^{\text {op }}}\left(Q^{\bullet}, A\right)\right)=0$ for $i>d$, the first two assertions follow.

Next, assume that $\operatorname{Ext}_{A^{\circ \mathrm{p}}}^{j}\left(\operatorname{Ext}_{A}^{i}(X, A), A\right)=0$ for $0 \leq j<i \leq d$. We have only to show that $\mathrm{H}^{-j}\left(\operatorname{Hom}_{A^{\circ \text { op }}}\left(Q^{\bullet}, A\right)\right)=0$ for $1 \leq j \leq d$. Note that $\operatorname{Hom}_{A^{\text {op }}}\left(Q^{\bullet}, A\right) \cong D\left(\sigma_{\leq d}\left(Q^{\bullet}\right)\right)$. For each $1 \leq i \leq d$ we have a distinguished triangle in $\mathcal{D}^{\mathrm{b}}\left(\bmod -A^{\mathrm{op}}\right)$

$$
\sigma_{\leq i-1}\left(Q^{\bullet}\right) \rightarrow \sigma_{\leq i}\left(Q^{\bullet}\right) \rightarrow \operatorname{Ext}_{A}^{i}(X, A)[-i] \rightarrow
$$

and hence a distinguished triangle in $\mathcal{D}^{\mathrm{b}}(\bmod -A)$

$$
D \operatorname{Ext}_{A}^{i}(X, A)[i] \rightarrow D\left(\sigma_{\leq i}\left(Q^{\bullet}\right)\right) \rightarrow D\left(\sigma_{\leq i-1}\left(Q^{\bullet}\right)\right) \rightarrow .
$$

We have $\mathrm{H}^{-j}\left(D \operatorname{Ext}_{A}^{i}(X, A)[i]\right) \cong \operatorname{Ext}_{A^{\text {op }}}^{i-j}\left(\operatorname{Ext}_{A}^{i}(X, A), A\right)=0$ for $1 \leq j \leq i \leq d$. Also, $\mathrm{H}^{-j}\left(D\left(\sigma_{\leq i}\left(Q^{\bullet}\right)\right)\right)=0$ for $j>i$. It follows by the long exact cohomology sequences that $\mathrm{H}^{-j}\left(D\left(\sigma_{\leq d}\left(Q^{\bullet}\right)\right)\right)=0$ for $1 \leq j \leq d$. 


\section{Finiteness of selfinjective dimension}

In this section, we will provide a necessary and sufficient condition for noetherian algebras to have finite selfinjective dimension.

Throughout the rest of this note, $(R, \mathfrak{m})$ is a commutative noetherian local ring and $A$ is a noetherian $R$-algebra, i.e., $A$ is a ring endowed with a ring homomorphism $R \rightarrow A$ whose image is contained in the center of $A$ and $A$ is finitely generated as an $R$-module. It should be noted that $A / \mathfrak{m} A$ is a finite dimensional algebra over a field $R / \mathfrak{m}$.

We denote by $\operatorname{Spec}(R)$ the set of prime ideals of $R$. For each $\mathfrak{p} \in \operatorname{Spec}(R)$ we denote by $(-)_{\mathfrak{p}}$ the localization at $\mathfrak{p}$ and for each $X \in \operatorname{Mod}-R$ we denote by $\operatorname{Supp}_{R}(X)$ the set of $\mathfrak{p} \in \operatorname{Spec}(R)$ with $X_{\mathfrak{p}} \neq 0$. Also, we denote by $\operatorname{dim} X$ the Krull dimension of $X \in \bmod -R$. We refer to [12] for standard commutative ring theory.

Lemma 3.1. We have $\operatorname{Ann}_{R}(S)=\mathfrak{m}$ for all simple $S \in \bmod -A$. In particular, $A / \operatorname{rad}(A) \in \bmod -A$ is semisimple.

Proof. Let $S \in \bmod -A$ be simple and $\mathfrak{p}=\operatorname{Ann}_{R}(S)$. Then we have an injective ring homomorphism $\varphi: R / \mathfrak{p} \rightarrow \operatorname{End}_{A}(S)$. Since $\operatorname{End}_{A}(S)$ is a division ring, $R / \mathfrak{p}$ is an integral domain and $\varphi$ can be extended to its quotient field $K$. Also, since $\operatorname{End}_{A}(S)$ is a subring of $\operatorname{End}_{R}(S)$, End ${ }_{A}(S)$ is finitely generated over $R / \mathfrak{p}$. Thus $K$ is finitely generated and hence integral over $R / \mathfrak{p}$, so that $K=R / \mathfrak{p}$ and $\mathfrak{p}=\mathfrak{m}$. It follows that $\mathfrak{m} A \subset \operatorname{rad}(A)$. Thus, since $A / \mathfrak{m} A$ is a finite dimensional algebra over a field $R / \mathfrak{m}, A / \operatorname{rad}(A)$ is an artinian ring and the assertion follows.

Lemma 3.2. For any injective $I \in \operatorname{Mod}-A$ the following hold.

(1) flat $\operatorname{dim} I \leq \operatorname{inj} \operatorname{dim} A^{\text {op }}$ and the equality holds if $I$ is an injective cogenerator.

(2) flat $\operatorname{dim} I \leq d$ if $\lim _{\longrightarrow} X_{\lambda} \cong I$ for some direct system $\left(\left\{X_{\lambda}\right\},\left\{f_{\mu}^{\lambda}\right\}\right)$ in mod-A over a directed set $\Lambda$ with $\hat{\mathrm{G}}$-dim $X_{\lambda} \leq d$ for all $\lambda \in \Lambda$.

Proof. (1) For any $M \in \bmod -A^{\text {op }}$ we have a functorial homomorphism

$$
\psi_{M}: I \otimes_{A} M \rightarrow \operatorname{Hom}_{A}\left(\operatorname{Hom}_{A^{\mathrm{op}}}(M, A), I\right), a \otimes x \mapsto(h \mapsto a h(x)) .
$$

Obviously, $\psi_{M}$ is an isomorphism if $M \in \mathcal{P}_{A^{\text {op }}}$. Thus, since both $I \otimes_{A}-$ and $\operatorname{Hom}_{A}\left(\operatorname{Hom}_{A^{\mathrm{op}}}(-, A), I\right)$ are right exact, $\psi_{M}$ is an isomorphism for all $M \in$ $\bmod -A^{\text {op }}$. It then follows that $\operatorname{Tor}_{i}^{A}(I, M) \cong \operatorname{Hom}_{A}\left(\operatorname{Ext}_{A^{\text {op }}}^{i}(M, A), I\right)$ for all $i \geq 0$ and $M \in \bmod -A^{\text {op }}$.

(2) For each $\lambda \in \Lambda$, since $\hat{\mathrm{G}}$ - $\operatorname{dim} X_{\lambda} \leq d$, by Corollary 2.6 we have a monomorphism $h_{\lambda}: X_{\lambda} \rightarrow Y_{\lambda}$ in mod- $A$ with proj $\operatorname{dim} Y_{\lambda} \leq n$. Set

$$
\tilde{Y}_{\lambda}=\Pi_{\mu \geq \lambda} Y_{\mu} \quad \text { and } \quad \tilde{h}_{\lambda}: X_{\lambda} \rightarrow \tilde{Y}_{\lambda}, x \mapsto\left(h_{\mu}\left(f_{\mu}^{\lambda}(x)\right)\right)_{\mu \geq \lambda}
$$

for each $\lambda \in \Lambda$, and denote by $g_{\mu}^{\lambda}: \tilde{Y}_{\lambda} \rightarrow \tilde{Y}_{\mu}$ the projection for each pair of $\lambda, \mu \in \Lambda$ with $\lambda \leq \mu$. It is not difficult to check that $\left(\left\{\tilde{Y}_{\lambda}\right\},\left\{g_{\mu}^{\lambda}\right\}\right)$ is a direct 
system in $\operatorname{Mod}-A$ and $\left\{\tilde{h}_{\lambda}\right\}$ is a monomorphism of direct systems. Note also that flat $\operatorname{dim} \tilde{Y}_{\lambda} \leq d$ for all $\lambda \in \Lambda$ and hence flat $\operatorname{dim} \lim \tilde{Y}_{\lambda} \leq d$. Now, since $\lim _{\longrightarrow} \tilde{h}_{\lambda}: \lim _{\longrightarrow} X_{\lambda} \rightarrow \lim _{\longrightarrow} \tilde{Y}_{\lambda}$ is a monomorphism (see e.g. [4, p. 287]), and since $\lim X_{\lambda} \cong I$, we have a split monomorphism $I \rightarrow \lim \tilde{Y}_{\lambda}$ and hence flat $\operatorname{dim} I \overrightarrow{\leq} d$.

Lemma 3.3. Let $E=\operatorname{Hom}_{R}\left(A, E_{R}(R / \mathfrak{m})\right)$ and $X_{n}=\left\{x \in E \mid \mathfrak{m}^{n} x=0\right\}$ for $n \geq 1$. Then the following hold.

(1) flat $\operatorname{dim} E_{A}=\operatorname{inj} \operatorname{dim} A^{\mathrm{op}}$.

(2) $E=\cup_{n \geq 1} X_{n}$ with the $X_{n} \in \operatorname{Mod}$-A of finite length.

Proof. (1) Since $\operatorname{Hom}_{A}\left(-, \operatorname{Hom}_{R}\left(A, E_{R}(R / \mathfrak{m})\right)\right) \cong \operatorname{Hom}_{R}\left(-, E_{R}(R / \mathfrak{m})\right)$, and since $E_{R}(R / \mathfrak{m}) \in \operatorname{Mod}-R$ is an injective cogenerator, it follows that $E \in \operatorname{Mod}-A$ is an injective cogenerator. Thus the assertion follows by Lemma 3.2(1).

(2) Since $A \in \operatorname{Mod}-R$ is finitely generated, $E$ is embedded in a finite direct sum of copies of $E_{R}(R / \mathfrak{m})$. Also, $E_{R}(R / \mathfrak{m})=\cup_{n \geq 1}\left\{x \in E_{R}(R / \mathfrak{m}) \mid \mathfrak{m}^{n} x=0\right\}$. It follows that $E=\cup_{n \geq 1} X_{n}$. Next, let $n \geq 1$. We have $\operatorname{Hom}_{R}\left(R / \mathfrak{m}^{n}, E\right) \stackrel{\sim}{\rightarrow} X_{n}$ and $\operatorname{Hom}_{R}\left(R / \mathfrak{m}^{n}, E\right) \cong \operatorname{Hom}_{R}\left(A / \mathfrak{m}^{n} A, E_{R}(R / \mathfrak{m})\right)$. Since $A / \mathfrak{m}^{n} A \in \operatorname{Mod}-R$ has finite length, so does $X_{n} \in \operatorname{Mod}-R$. Thus $X_{n} \in \operatorname{Mod}-R$ is both noetherian and artinian, so is $X_{n} \in \operatorname{Mod}-A$.

Lemma 3.4. Assume that $\operatorname{inj} \operatorname{dim} A=d<\infty$. Then the following hold.

(1) inj $\operatorname{dim} A_{\mathfrak{p}}<d$ for all non-maximal $\mathfrak{p} \in \operatorname{Supp}_{R}(A)$.

(2) The last non-zero term of a minimal injective resolution of $A$ in Mod- $A$ is a finite direct sum of indecomposable submodules with nonzero socle.

Proof. Let $A \rightarrow I^{\bullet}$ be a minimal injective resolution in Mod- $A$.

(1) Suppose to the contrary that $I^{d}$ contains an indecomposable direct summand $I$ with $I_{\mathfrak{p}} \neq 0$ for some non-maximal $\mathfrak{p} \in \operatorname{Supp}_{R}(A)$. Take a finitely generated nonzero submodule $X$ of $I$. Note that $X$ does not contain a simple submodule and there exists an $X$-regular element $x \in \mathfrak{m}$. Thus, applying $\operatorname{Hom}_{A}(-, A)$ to the exact sequence $0 \rightarrow X \stackrel{x}{\rightarrow} X$, we have an exact sequence $\operatorname{Ext}_{A}^{d}(X, A) \stackrel{x}{\rightarrow} \operatorname{Ext}_{A}^{d}(X, A) \rightarrow 0$, so that by Nakayama lemma $\operatorname{Ext}_{A}^{d}(X, A)=0$, a contradiction.

(2) It follows by (1) above that $I^{d}$ has an essential socle. Also, by Lemma 3.1 there are only a finite number of non-isomorphic simple modules in mod- $A$. Let $S_{1}, S_{2}, \cdots, S_{n} \in \bmod -A$ be the non-isomorphic simple modules. Note that every $\operatorname{Ext}_{A}^{d}\left(S_{i}, A\right)$ is finite dimensional over $R / \mathfrak{m}$. Denote by $\mu_{i}$ the dimension of $\operatorname{Ext}_{A}^{d}\left(S_{i}, A\right)$ over $\operatorname{End}_{A}\left(S_{i}\right)$ for each $1 \leq i \leq n$. Then $I^{d} \cong \oplus_{i=1}^{n} E_{A}\left(S_{i}\right)^{\mu_{i}}$ with $\sum_{i=1}^{n} \mu_{i}<\infty$.

Definition 3.5. We say that $A$ satisfies the condition $(\mathrm{G})$ if the following equivalent conditions are satisfied (cf. Lemma 3.1):

(1) $\hat{\mathrm{G}}$ - $\operatorname{dim} X<\infty$ for all simple $X \in \bmod -A$. 
(2) $\hat{\mathrm{G}}-\operatorname{dim} A / \operatorname{rad}(A)<\infty$.

Lemma 3.6. Assume that $A$ satisfies the condition $(G)$. Then, setting $d=$ $\hat{\mathrm{G}}-\operatorname{dim} A / \operatorname{rad}(A)$, we have inj $\operatorname{dim} A^{\mathrm{op}} \leq d$.

Proof. According to Lemma 2.7(1), it follows by induction on the Loewy length that $\mathrm{G}$-dim $X \leq d$ for all $X \in \bmod -A$ of finite length. The assertion follows by Lemmas 3.2(2) and 3.3.

Theorem 3.7. Let $(R, \mathfrak{m})$ be a commutative noetherian local ring and $A$ a noetherian $R$-algebra. Then the following are equivalent.

(1) $\operatorname{inj} \operatorname{dim} A=\operatorname{inj} \operatorname{dim} A^{\mathrm{op}}<\infty$.

(2) $A_{\mathfrak{p}}$ satisfies the condition $(G)$ for all $\mathfrak{p} \in \operatorname{Supp}_{R}(A)$.

Proof. (1) $\Rightarrow(2)$. For any $\mathfrak{p} \in \operatorname{Supp}_{R}(A)$, by [14, Lemma A] and Lemma 3.4 we have $\operatorname{inj} \operatorname{dim} A_{\mathfrak{p}}=\operatorname{inj} \operatorname{dim} A_{\mathfrak{p}}^{\text {op }}<\infty$, so that by [9, Lemma 6$]$ every $X \in \bmod -A_{\mathfrak{p}}$ has finite Gorenstein dimension.

$(2) \Rightarrow(1)$. Let $d=\hat{\mathrm{G}}-\operatorname{dim} A / \operatorname{rad}(A)$. Then by Lemma $3.6 \operatorname{inj} \operatorname{dim} A^{\mathrm{op}} \leq d$. We claim that $\operatorname{inj} \operatorname{dim} A<\infty$. We will make use of induction on $\operatorname{dim} A$. Consider first the case where $\operatorname{Supp}_{R}(A)=\{\mathfrak{m}\}$. Then $A$ is an artinian ring and hence $\operatorname{Ext}_{A}^{d+1}(A / \operatorname{rad}(A), A)=0$ implies $\operatorname{inj} \operatorname{dim} A \leq d$. Next, assume that $\operatorname{Supp}_{R}(A) \neq\{\mathfrak{m}\}$ and that $\operatorname{inj} \operatorname{dim} A_{\mathfrak{p}}<\infty$ for all non-maximal $\mathfrak{p} \in$ $\operatorname{Supp}_{R}(A)$. Taking a minimal injective resolution $A \rightarrow I^{\bullet}$ in Mod- $A$, we have $\operatorname{Hom}_{A}\left(A / \operatorname{rad}(A), I^{d+1}\right) \cong \operatorname{Ext}_{A}^{d+1}(A / \operatorname{rad}(A), A)=0$ and $\operatorname{Hom}_{R}\left(R / \mathfrak{m}, I^{d+1}\right) \cong$ $\operatorname{Hom}_{A}\left(A / \mathfrak{m} A, I^{d+1}\right)=0$. Also, for any non-maximal $\mathfrak{p} \in \operatorname{Supp}_{R}(A)$, by [14, Lemma A] and Lemma 3.4(1) we have inj $\operatorname{dim} A_{\mathfrak{p}}<d$ and hence $I_{\mathfrak{p}}^{d+1}=0$. It follows that $I^{d+1}=0$ and $\operatorname{inj} \operatorname{dim} A \leq d$.

\section{Gorenstein algebras}

In this section, we will be mainly concerned with the case where inj $\operatorname{dim} A=$ $\operatorname{inj} \operatorname{dim} A^{\text {op }}=\operatorname{depth} A$, i.e., $A$ is a Gorenstein algebra in the sense of Goto and Nishida [7] and characterize local noetherian algebras of finite selfinjective dimension in terms of weak Gorenstein dimension.

We set $S=A / \operatorname{rad}(A)$ and denote by depth $X$ the depth of $X \in \bmod -R$.

Lemma 4.1. For any nonzero $X \in \bmod -A$ we have

$$
\operatorname{depth} X=\inf \left\{i \mid \operatorname{Ext}_{A}^{i}(S, X) \neq 0\right\} .
$$

Proof. We will make use of induction on $d=\operatorname{depth} X$. Consider first the case where $d=0$. Then $\operatorname{Hom}_{A}(A / \mathfrak{m} A, X) \cong \operatorname{Hom}_{R}(R / \mathfrak{m}, X) \neq 0$, so that $X$ has a nonzero socle and $\operatorname{Hom}_{A}(S, X) \neq 0$. Next, assume that $d \geq 1$ and take an $X$-regular element $x \in \mathfrak{m}$. Then depth $X / x X=d-1$ and by induction hypothesis $\inf \left\{i \mid \operatorname{Ext}_{A}^{i}(S, X / x X) \neq 0\right\}=d-1$. Since $\operatorname{Hom}_{A}(A / \mathfrak{m} A, X) \cong$ 
$\operatorname{Hom}_{R}(R / \mathfrak{m}, X)=0, \operatorname{Hom}_{A}(S, X)=0$. Also, $x \operatorname{Ext}_{A}^{i}(S, X)=0$ for all $i \geq 0$ because $\mathfrak{m} S=0$. Thus, applying $\operatorname{Hom}_{A}(S,-)$ to the exact sequence

$$
0 \rightarrow X \stackrel{x}{\rightarrow} X \rightarrow X / x X \rightarrow 0
$$

we have exact sequences

$$
0 \rightarrow \operatorname{Ext}_{A}^{i-1}(S, X) \rightarrow \operatorname{Ext}_{A}^{i-1}(S, X / x X) \rightarrow \operatorname{Ext}_{A}^{i}(S, X) \rightarrow 0
$$

for $i \geq 1$, so that $\operatorname{Ext}_{A}^{i-1}(S, X / x X)=0$ implies $\operatorname{Ext}_{A}^{i}(S, X)=0$ for $1 \leq i<d$ and $\operatorname{Ext}_{A}^{d}(S, X) \cong \operatorname{Ext}_{A}^{d-1}(S, X / x X) \neq 0$.

Lemma 4.2. If inj $\operatorname{dim} A=\operatorname{depth} A=d<\infty$ then the following hold.

(1) A is Cohen-Macaulay as an R-module.

(2) $\hat{\mathrm{G}}-\operatorname{dim} X=d$ for all simple $X \in \bmod -A$ with $\operatorname{Ext}_{A}^{d}(X, A) \neq 0$.

(3) $\operatorname{inj} \operatorname{dim} A^{\mathrm{op}}=d$ if $\operatorname{Ext}_{A}^{d}(X, A) \neq 0$ for all simple $X \in \bmod -A$.

Proof. (1) It follows by Lemma 3.4(1) that $\operatorname{dim} A \leq \operatorname{inj} \operatorname{dim} A$. Thus $\operatorname{dim} A \leq$ depth $A$ and hence $\operatorname{dim} A=\operatorname{depth} A$.

(2) Let $X \in \bmod -A$ be simple with $\operatorname{Ext}_{A}^{d}(X, A) \neq 0$. Set $L=\operatorname{Ext}_{A}^{d}(X, A) \in$ $\bmod -A^{\text {op }}$. Then $D X \cong L[-d]$ in $\mathcal{D}\left(\operatorname{Mod}-A^{\text {op }}\right)$ and, since $L \neq 0, \eta_{X} \neq 0$ in $\mathcal{D}(\operatorname{Mod}-A)$. Also, for any $i<d$, by Lemma $4.1 \operatorname{Ext}_{A^{\text {op }}}^{i}(S, A)=0$ and it follows by induction on the Loewy length that $\operatorname{Ext}_{A^{\text {op }}}^{i}(-, A)$ vanishes on $\bmod -(A / \mathfrak{m} A)^{\mathrm{op}}$. Thus $\operatorname{Ext}_{A^{\mathrm{op}}}^{i}(L, A)=0$ for $i<d$. Now, take a projective resolution $P^{\bullet} \rightarrow X$ in mod- $A$ and a projective resolution $Q^{\bullet} \rightarrow L$ in mod- $A^{\text {op }}$. Then $D^{2} X \cong \sigma_{\geq d}^{\prime}\left(\operatorname{Hom}_{A^{\text {op }}}\left(Q^{\bullet}, A\right)\right)[d]$ in $\mathcal{D}(\operatorname{Mod}-A)$ and $\eta_{X}$ is given by a cochain map

$$
\eta: P^{\bullet} \rightarrow \sigma_{\geq d}^{\prime}\left(\operatorname{Hom}_{A^{\text {op }}}\left(Q^{\bullet}, A\right)\right)[d]
$$

Since $\eta$ is not homotopic to zero, $\mathrm{H}^{0}(\eta) \neq 0$. Thus, since $X$ is simple, $\mathrm{H}^{0}(\eta)$ is a monomorphism. It follows that $\hat{\mathrm{G}}$-dim $X<\infty$ and hence $\hat{\mathrm{G}}$-dim $X=d$.

(3) According to (2) above, we have $\hat{\mathrm{G}}$ - $\operatorname{dim} S_{A}=d$ and by Lemma 3.6 inj $\operatorname{dim} A^{\text {op }} \leq d$, so that by $\left[14\right.$, Lemma A] inj $\operatorname{dim} A^{\text {op }}=d$.

Lemma 4.3. We have inj $\operatorname{dim} A=\operatorname{inj} \operatorname{dim} A^{\mathrm{op}}=d$ if $\hat{\mathrm{G}}-\operatorname{dim} S_{A}=\operatorname{depth} A=$ $d<\infty$.

Proof. By Lemma 3.6 inj $\operatorname{dim} A^{\text {op }} \leq d$. Also, since $\operatorname{Ext}_{A}^{d}(S, A) \neq 0, \operatorname{inj} \operatorname{dim} A \geq$ $d$. Thus, according to [14, Lemma A], it suffices to show that inj $\operatorname{dim} A \leq d$.

We divide the proof into several steps.

Claim 1. For any $X \in \bmod -A / \mathfrak{m} A$ and $M \in \bmod -(A / \mathfrak{m} A)^{\text {op }}$ we have a bifunctorial isomorphism $\operatorname{Hom}_{A^{\text {op }}}\left(M, \operatorname{Ext}_{A}^{d}(X, A)\right) \cong \operatorname{Hom}_{A}\left(X, \operatorname{Ext}_{A^{\text {op }}}^{d}(M, A)\right)$, and $X \stackrel{\sim}{\rightarrow} \operatorname{Ext}_{A}^{d}{ }_{\text {op }}\left(\operatorname{Ext}_{A}^{d}(X, A), A\right)$ canonically for all $X \in \bmod -A / \mathfrak{m} A$. 
Proof. Note that by Lemma 4.1 we have $\operatorname{Ext}_{A}^{i}(S, A)=0$ for $i \neq d$. It follows by induction on the Loewy length that $\operatorname{Ext}_{A}^{i}(-, A)$ vanishes on mod- $A / \mathfrak{m} A$ for $i \neq d$ and $D X \cong \operatorname{Ext}_{A}^{d}(X, A)[-d]$ in $\mathcal{D}\left(\operatorname{Mod}-A^{\text {op }}\right)$ for all $X \in \bmod -A / \mathfrak{m} A$. Similarly, since inj $\operatorname{dim} A^{\text {op }} \leq d$, by Lemma 4.1 we have $\operatorname{Ext}_{A^{\text {op }}}^{i}(S, A)=0$ for $i \neq d$, so that $\operatorname{Ext}_{A \text { op }}^{i}(-, A)$ vanishes on $\bmod -(A / \mathfrak{m} A)^{\text {op }}$ for $i \neq d$ and $D M \cong \operatorname{Ext}_{A^{\text {op }}}^{d}(M, A)[-d]$ in $\mathcal{D}(\operatorname{Mod}-A)$ for all $M \in \bmod -(A / \mathfrak{m} A)^{\text {op }}$. Thus we have bifunctorial isomorphisms

$$
\begin{aligned}
\operatorname{Hom}_{A^{\mathrm{op}}}\left(M, \operatorname{Ext}_{A}^{d}(X, A)\right) & \cong \operatorname{Hom}_{\mathcal{D}\left(\operatorname{Mod}-A^{\mathrm{op}}\right)}(M, D X[d]) \\
& \cong \operatorname{Hom}_{\mathcal{D}(\operatorname{Mod}-A)}(X, D M[d]) \\
& \cong \operatorname{Hom}_{A}\left(X, \operatorname{Ext}_{A^{\mathrm{op}}}^{d}(M, A)\right)
\end{aligned}
$$

for $X \in \bmod -A / \mathfrak{m} A$ and $M \in \bmod -(A / \mathfrak{m} A)^{\mathrm{op}}$. Also, for any $X \in \bmod -A / \mathfrak{m} A$, since $\operatorname{inj} \operatorname{dim} A^{\text {op }} \leq d$, by Lemma 1.8 together with Remark 1.9 we have G-dim $X<\infty$ and hence a functorial isomorphism

$$
X \stackrel{\sim}{\rightarrow} \operatorname{Ext}_{A^{\text {op }}}^{d}\left(\operatorname{Ext}_{A}^{d}(X, A), A\right)
$$

Claim 2. $L=\operatorname{Ext}_{A}^{d}(A / \mathfrak{m} A, A)$ is an injective cogenerator for $\bmod -(A / \mathfrak{m} A)^{\mathrm{op}}$.

Proof. Since by Claim $1 \mathcal{P}_{A / \mathfrak{m} A} \stackrel{\sim}{\rightarrow} \operatorname{add}(L)$, the number of non-isomorphic indecomposable direct summands of $L$ coincides with that of non-isomorphic simple modules. Thus we have only to show that $L$ is injective in $\bmod -(A / \mathfrak{m} A)^{\text {op }}$. Let $f: L \rightarrow M$ be a monomorphism in $\bmod -(A / \mathfrak{m} A)^{\mathrm{op}}$. Then $\operatorname{Ext}_{A^{\mathrm{op}}}^{d}(f, A)$ is an epimorphism and, since by Claim $1 A / \mathfrak{m} A \stackrel{\sim}{\rightarrow} \operatorname{Ext}_{A^{\circ \mathrm{p}}}^{d}(L, A)$, splits. Thus $\operatorname{Ext}_{A}^{d}\left(\operatorname{Ext}_{A^{\text {op }}}^{d}(f, A), A\right)$ is a split monomorphism, so is $f$ because by Claim 1 $L \stackrel{\sim}{\rightarrow} \operatorname{Ext}_{A}^{d}\left(\operatorname{Ext}_{A^{\circ \mathrm{p}}}^{d}(L, A), A\right)$.

Claim 3. $\operatorname{Ext}_{A}^{d}(X, A) \in \bmod -A^{\text {op }}$ is simple with G-dim $\operatorname{Ext}_{A}^{d}(X, A)=d$ for all simple $X \in \bmod -A$.

Proof. Note that by Lemma $3.1 X \in \bmod -A / \mathfrak{m} A$ and hence $\operatorname{Ext}_{A}^{d}(X, A) \in$ $\bmod -(A / \mathfrak{m} A)^{\mathrm{op}}$. Let $0 \rightarrow M \rightarrow \operatorname{Ext}_{A}^{d}(X, A) \rightarrow N \rightarrow 0$ be an exact sequence in $\bmod -(A / \mathfrak{m} A)^{\mathrm{op}}$. Then $0 \rightarrow \operatorname{Ext}_{A^{\text {op }}}^{d}(N, A) \rightarrow X \rightarrow \operatorname{Ext}_{A^{\text {op }}}^{d}(M, A) \rightarrow 0$ is an exact sequence in mod- $A / \mathfrak{m} A$ with $X$ simple, so that either $\operatorname{Ext}_{A^{\text {op }}}^{d}(M, A)=0$ or $\operatorname{Ext}_{A^{\text {op }}}^{d}(N, A)=0$. On the other hand, since by Claim 1

$$
\begin{aligned}
\operatorname{Ext}_{A^{\text {op }}}^{d}(M, A) & \cong \operatorname{Hom}_{A}\left(A / \mathfrak{m} A, \operatorname{Ext}_{A^{\text {op }}}^{d}(M, A)\right) \\
& \cong \operatorname{Hom}_{A^{\text {op }}}(M, L),
\end{aligned}
$$

by Claim $2 \operatorname{Ext}_{A^{\text {op }}}^{d}(M, A)=0$ implies $M=0$. Similarly, $\operatorname{Ext}_{A^{\text {op }}}^{d}(N, A)=0$ implies $N=0$. It follows that $\operatorname{Ext}_{A}^{d}(X, A)$ is simple. Finally, by Remark 1.7(2) G-dim $\operatorname{Ext}_{A}^{d}(X, A)<\infty$ and hence G-dim $\operatorname{Ext}_{A}^{d}(X, A)=d$.

Now, it follows by Claims 1 and 3 that $A^{\text {op }}$ satisfies the condition $(\mathrm{G})$. Thus by Lemma $3.6 \operatorname{inj} \operatorname{dim} A \leq d$. 
Lemma 4.4. For any $X \in \operatorname{Mod}-A$ with flat $\operatorname{dim} X=n<\infty$ and $Y \in \bmod -A$ with $\inf \left\{i \mid \operatorname{Ext}_{A}^{i}(Y, A) \neq 0\right\}=d<\infty$ we have $\operatorname{Hom}_{\mathcal{D}(\operatorname{Mod}-A)}(Y, X[i])=0$ for $i<d-n$ and $\operatorname{Hom}_{\mathcal{D}(\operatorname{Mod}-A)}(Y, X[d-n]) \cong \operatorname{Tor}_{n}^{A}\left(X, \operatorname{Ext}_{A}^{d}(Y, A)\right)$.

Proof. Take a flat resolution $F^{\bullet} \rightarrow X$ in Mod- $A$ with $F^{-i}=0$ for $i>n$ and a projective resolution $P^{\bullet} \rightarrow Y$ in $\bmod -A$. Set $Q^{\bullet}=\sigma_{\geq d}^{\prime}\left(\operatorname{Hom}_{A}^{\bullet}\left(P^{\bullet}, A\right)\right)$. Then

$$
\begin{aligned}
\operatorname{Hom}_{\mathcal{D}(\operatorname{Mod}-A)}(Y, X[i]) & \cong \operatorname{Hom}_{\mathcal{K}(\operatorname{Mod}-A)}\left(P^{\bullet}, F^{\bullet}[i]\right) \\
& \cong \mathrm{H}^{i}\left(\operatorname{Hom}_{A}^{\bullet}\left(P^{\bullet}, F^{\bullet}\right)\right) \\
& \cong \mathrm{H}^{i}\left(F^{\bullet} \otimes_{A}^{\bullet} \operatorname{Hom}_{A}^{\bullet}\left(P^{\bullet}, A\right)\right) \\
& \cong \mathrm{H}^{i}\left(F^{\bullet} \otimes_{A}^{\bullet} Q^{\bullet}\right)
\end{aligned}
$$

for all $i \in \mathbb{Z}$. Also, $\mathrm{H}^{i}\left(F^{\bullet} \otimes_{A}^{\bullet} Q^{\bullet}\right)=0$ for $i<d-n$ and

$$
\begin{aligned}
\mathrm{H}^{d-n}\left(F^{\bullet} \otimes_{A}^{\bullet} Q^{\bullet}\right) & \cong \mathrm{Z}^{-n}\left(F^{\bullet} \otimes_{A}^{\bullet} Q^{d}\right) \cap \mathrm{Z}^{d}\left(F^{-n} \otimes_{A}^{\bullet} Q^{\bullet}\right) \\
& \cong \mathrm{Z}^{-n}\left(F^{\bullet} \otimes_{A}^{\bullet} \operatorname{Ext}_{A}^{d}(Y, A)\right) \\
& \cong \operatorname{Tor}_{n}^{A}\left(X, \operatorname{Ext}_{A}^{d}(Y, A)\right) .
\end{aligned}
$$

Throughout the rest of this note, we assume that $A$ is a local ring, i.e., $S$ is a division ring.

Note that $S \in \bmod -A$ is a unique simple module up to isomorphism and that every $X \in \bmod -A$ admits a minimal projective resolution.

Proposition 4.5. For any $X \in \bmod -A$ with $\operatorname{proj} \operatorname{dim} X<\infty$ we have

$$
\text { proj } \operatorname{dim} X+\operatorname{depth} X=\operatorname{depth} A \text {. }
$$

Proof. Let $n=\operatorname{proj} \operatorname{dim} X$. Since $X$ admits a minimal projective resolution in $\bmod -A$, we have $\operatorname{Tor}_{n}^{A}(X, S) \neq 0$. Let $L=\operatorname{Ext}_{A}^{d}(S, A) \in \bmod -A^{\text {op }}$ with $d=$ depth $A$. Note that $L \in \bmod -A^{\text {op }}$ has finite length. Thus, since $\operatorname{Tor}_{n}^{A}(X, S) \neq 0$, and since $\operatorname{Tor}_{n}^{A}(X,-)$ is left exact, we have $\operatorname{Tor}_{n}^{A}(X, L) \neq 0$ and the assertion follows by Lemma 4.4 .

Corollary 4.6. We have $\operatorname{depth} A=d$ if $\hat{\mathrm{G}}-\operatorname{dim} S_{A}=d<\infty$.

Proof. Obviously, depth $A \leq d$. On the other hand, by Corollary 2.6 there exists $X \in \bmod -A$ with proj $\operatorname{dim} X=d$ and by Proposition $4.5 \operatorname{depth} A \geq d$.

Theorem 4.7. Let $R$ be a commutative noetherian local ring and $A$ a local noetherian $R$-algebra with $S=A / \operatorname{rad}(A)$. Then for any $d \geq 0$ the following are equivalent.

(1) $\operatorname{inj} \operatorname{dim} A=\operatorname{inj} \operatorname{dim} A^{\mathrm{op}}=d$.

(2) $\operatorname{inj} \operatorname{dim} A=\operatorname{depth} A=d$. 
(3) $\hat{\mathrm{G}}-\operatorname{dim} S_{A}=d$.

Proof. (1) $\Rightarrow(2)$. By Theorem $3.7 \hat{\mathrm{G}}$ - $\operatorname{dim} S_{A}<\infty$, so that by Lemma 3.4(2) G-dim $S_{A}=d$ and hence by Corollary $4.6 \operatorname{depth} A=d$.

$(2) \Rightarrow(3)$. By Lemma 4.2(2).

$(3) \Rightarrow(1)$. By Lemma 4.3 together with Corollary 4.6.

Proposition 4.8. The following hold.

(1) $E_{A}(S) \cong \operatorname{Hom}_{R}\left(A, E_{R}(R / \mathfrak{m})\right)$.

(2) If $\operatorname{inj} \operatorname{dim} A=\operatorname{inj} \operatorname{dim} A^{\mathrm{op}}=d<\infty$ then $I^{d} \cong \operatorname{Hom}_{R}\left(A, E_{R}(R / \mathfrak{m})\right)$ in a minimal injective resolution $A \rightarrow I^{\bullet}$ in $\operatorname{Mod}-A$.

Proof. (1) Since $S \in \bmod -R$ has finite length, $\operatorname{Hom}_{R}\left(S, E_{R}(R / \mathfrak{m})\right) \cong S$ in $\bmod -S$. Also, $\operatorname{Hom}_{A}\left(S, \operatorname{Hom}_{R}\left(A, E_{R}(R / \mathfrak{m})\right)\right) \cong \operatorname{Hom}_{R}\left(S, E_{R}(R / \mathfrak{m})\right)$ in mod- $S$. It follows that $\operatorname{Hom}_{R}\left(A, E_{R}(R / \mathfrak{m})\right) \in \bmod -A$ has a simple socle.

(2) We have $\operatorname{Hom}_{A}\left(S, I^{d}\right) \cong \operatorname{Ext}_{A}^{d}(S, A)$ in mod-S. Also, we have seen in the proof of Lemma 4.3 that $\operatorname{Ext}_{A}^{d}(S, A) \cong S$ in mod- $A^{\mathrm{op}}$. Thus $\operatorname{Hom}_{A}\left(S, I^{d}\right)$ and $S$ have the same dimension as vector spaces over $R / \mathfrak{m}$ and hence $\operatorname{Hom}_{A}\left(S, I^{d}\right) \cong S$ in mod- $S$, so that $I^{d}$ has a simple socle.

Example 4.9. Even if inj $\operatorname{dim} A=\operatorname{inj} \operatorname{dim} A^{\text {op }}<\infty$, it may happen that $A$ is not Cohen-Macaulay as an $R$-module. For instance, let $R$ be a Gorenstein local ring with $\operatorname{dim} R \geq 1$ and set

$$
A=\left(\begin{array}{cc}
R & R / x R \\
0 & R / x R
\end{array}\right)
$$

with $x \in \mathfrak{m}$ a regular element. Then $A$ is not Cohen-Macaulay as an $R$-module but inj $\operatorname{dim} A=\operatorname{inj} \operatorname{dim} A^{\mathrm{op}}<\infty$ (see [1, Example 4.7]).

Example 4.10. Even if $A$ is Cohen-Macaulay as an $R$-module and $\operatorname{inj} \operatorname{dim} A=$ $\operatorname{inj} \operatorname{dim} A^{\text {op }}<\infty$, it may happen that $\operatorname{inj} \operatorname{dim} A \neq \operatorname{depth} A$. For instance, let $R$ be a Gorenstein local ring with $\operatorname{dim} R=d$ and set

$$
A=\left(\begin{array}{cc}
R & R \\
0 & R
\end{array}\right) .
$$

Then $A$ is a Cohen-Macaulay $R$-module with $\operatorname{depth} A=d \operatorname{but} \operatorname{inj} \operatorname{dim} A=$ $\operatorname{inj} \operatorname{dim} A^{\mathrm{op}}=d+1$.

Example 4.11. Even if $\operatorname{inj} \operatorname{dim} A=\operatorname{inj} \operatorname{dim} A^{\text {op }}=\operatorname{depth} A=d<\infty$, it may happen that $I^{d} ¥ \operatorname{Hom}_{R}\left(A, E_{R}(R / \mathfrak{m})\right)$ for a minimal injective resolution $A \rightarrow I^{\bullet}$ in Mod- $A$. For instance, let $R$ be a Gorenstein local ring with $\operatorname{dim} R=$ $d$ and $A$ a free $R$-module with a basis $\left\{e_{i j}\right\}_{1 \leq i, j \leq 3}$. Define a multiplication on $A$ subject to the following axioms: (A1) $e_{i j} e_{k l}=0$ unless $j=k$; (A2) $e_{i i} e_{i j}=e_{i j}=e_{i j} e_{j j}$ for all $i, j$; (A3) $e_{12} e_{21}=e_{11}$ and $e_{21} e_{12}=e_{22}$; and (A4) $e_{i 3} e_{3 j}=e_{3 j} e_{i 3}=0$ for all $i, j \neq 3$. Set $e_{i}=e_{i i}$ for all $i$. Then $A$ is an $R$ algebra with $1=e_{1}+e_{2}+e_{3}$ and Cohen-Macaulay as an $R$-module. Also, 
setting $\Omega=\operatorname{Hom}_{R}(A, R)$, we have $e_{1} A \cong e_{2} A \cong e_{3} \Omega$ and $e_{1} \Omega \cong e_{2} \Omega \cong e_{3} A$. It follows that inj $\operatorname{dim} A=\operatorname{inj} \operatorname{dim} A^{\text {op }}=d$ but $I^{d} \cong \operatorname{Hom}_{R}\left(\Omega, E_{R}(R / \mathfrak{m})\right) \nsucceq$ $\operatorname{Hom}_{R}\left(A, E_{R}(R / \mathfrak{m})\right)$ in Mod- $A$.

\section{References}

[1] H. Abe and M. Hoshino, Derived equivalences and Gorenstein algebras, J. Pure Appl. Algebra 211, 55-69 (2007).

[2] M. Auslander and M. Bridger, Stable module theory, Mem. Amer. Math. Soc., 94, Amer. Math. Soc., Providence, R.I., 1969.

[3] M. Bökstedt and A. Neeman, Homotopy limits in triangulated categories, Compositio Math. 86 (1993), no. 2, 209-234.

[4] N. Bourbaki, Elements of mathematics. Algebra, Part I: Chapters 1-3. Translated from the French. Hermann, Paris; Addison-Wesley Publishing Co., Reading Mass., 1974.

[5] S. U. Chase, Direct products of modules, Trans. Amer. Math. Soc. 97 (1960), 457-473.

[6] L. W. Christensen, A. Frankild and H. Holm, On Gorenstein projective, injective and flat dimensions - a functorial description with applications, J. Algebra 302 (2006), no. 1, 231-279.

[7] S. Goto and K. Nishida, Towards a theory of Bass numbers with application to Gorenstein algebras, Colloquium Math. 91 (2002), 191-253.

[8] R. Hartshorne, Residues and duality, Lecture Notes in Math., 20, Springer, Berlin, 1966.

[9] M. Hoshino, Algebras of finite self-injective dimension, Proc. Amer. Math. Soc. 112 (1991), 619-622.

[10] M. Hoshino and H. Koga, Zaks' lemma for coherent rings, preprint.

[11] Y. Kato, On derived equivalent coherent rings, Comm. Algebra 30 (2002), no. $9,4437-4454$.

[12] H. Matsumura, Commutative Ring Theory (M. Reid, Trans.), Cambridge Univ. Press, Cambridge, 1986 (original work in Japanese).

[13] J. L. Verdier, Catégories dérivées, état 0, in: Cohomologie étale, 262-311, Lecture Notes in Math., 569, Springer, Berlin, 1977.

[14] A. Zaks, Injective dimension of semi-primary rings, J. Algebra 13 (1969), $73-86$. 
Institute of Mathematics, University of Tsukuba, Ibaraki, 305-8571, Japan

E-mail address: hoshino@math.tsukuba.ac.jp

Institute of Mathematics, University of Tsukuba, Ibaraki, 305-8571, Japan

E-mail address: koga@math.tsukuba.ac.jp 